\title{
Evaluation of Force-Based Frame Element Response Sensitivity Formulations
}

\author{
Michael H. Scott, A.M.ASCE${ }^{1}$
}

\begin{abstract}
The direct differentiation method (DDM) has been shown to be an accurate and efficient approach to computing the sensitivity of structural response to uncertain parameters of constitutive models and finite-element formulations. Although it is well-known that the DDM should be consistent with the numerical time stepping procedure at the structural level, it is possible for element-level numerical instabilities to arise when the response sensitivity equations are inconsistent with the equations that govern the element response. Two existing formulations of DDM force-based element response sensitivity are shown to be mathematically equivalent in exact arithmetic; however, only one is consistent with the force-based response equations and possesses a low condition number for finite arithmetic. On the other hand, the inconsistent formulation has a high condition number that is equal to the product of the largest singular values of the section and element stiffness matrices. Representative examples show that the high condition number of the inconsistent formulation is innocuous for sensitivity with respect to section-level parameters but can lead to round off errors for sensitivity with respect to element-level geometric parameters. DOI: 10.1061/(ASCE)ST.1943-541X.0000447. () 2012 American Society of Civil Engineers.
\end{abstract}

CE Database subject headings: Sensitivity analysis; Frames; Reliability; Optimization; Numerical analysis.

Author keywords: Sensitivity analysis; Frame elements; Reliability; Optimization; Numerical analysis.

\section{Introduction}

Response sensitivity analysis is an essential component to gradientbased structural engineering applications such as reliability, optimization, and system identification in which structural response depends on uncertain material, load, and geometric parameters. Two fundamental approaches to response sensitivity analysis are available. In the finite difference method, analyses are repeated with perturbed parameters to approximate gradients of the structural response. Although this approach is easy to implement, its accuracy is subject to the magnitude of the parameter perturbations, and it can be inefficient to reanalyze the structure once for each uncertain parameter. On the other hand, the direct differentiation method (DDM) is difficult to implement but requires neither parameter perturbations nor reanalysis. In the DDM, the governing equations of structural equilibrium, compatibility, and equilibrium are differentiated analytically with respect to the uncertain parameters and programmed as part of the finite-element core (Choi and Santos 1987; Tsay and Arora 1990; Kleiber et al. 1997).

Considerable research has been devoted to making finiteelement formulations and constitutive models available for use with the DDM and its accompanying gradient-based applications. A sampling of this research includes the application of the DDM to composite beams (Zona et al. 2005), steel moment resisting frames (Gong et al. 2006), reinforced concrete frames (Habibi and Moharrami 2010), and soil constitutive models (Fellin and Ostermann 2006). Further extensions of the DDM have been

\footnotetext{
${ }^{1}$ Associate Professor, School of Civil and Construction Engineering, Oregon State Univ., Corvallis, OR 97331. E-mail michael.scott@ oregonstate.edu

Note. This manuscript was submitted on October 15, 2010; approved on May 20, 2011; published online on May 25, 2011. Discussion period open until June 1, 2012; separate discussions must be submitted for individual papers. This paper is part of the Journal of Structural Engineering, Vol. 138, No. 1, January 1, 2012. CASCE, ISSN 0733-9445/2012/1$72-80 / \$ 25.00$.
}

developed for handling multipoint constraints (Gu et al. 2009), follower loads (Pajot and Maute 2006), and large displacements by using the corotational formulation (Scott and Filippou 2007). In addition, the DDM has been extended for computing the second derivative of structural response (Bebamzadeh and Haukaas 2008).

In the aforementioned references, accuracy of the DDM was shown by convergence of finite difference calculations of decreasing parameter perturbations to the exact derivative, whereas efficiency is achieved because the DDM requires repeated use of a factorized stiffness matrix instead of full reanalysis for every parameter. However, many of the numerical issues associated with computing finite-element response are also relevant in computing DDM response sensitivity. Similar to the use of a numerically consistent tangent matrix, derivatives must be measured with respect to the space and time discretized equations rather than continuous rate equations (Conte et al. 2003). Furthermore, the convergence of gradient-based algorithms is improved when the DDM is applied to smooth constitutive models (Barbato and Conte 2006). Specialized approaches to the DDM have been developed to reduce the computational cost associated with path-dependent problems (Haukaas 2006).

With accuracy and efficiency of the DDM well-established and selected computational issues addressed, the ramifications of a sensitivity formulation that is inconsistent with the underlying finiteelement response has not been addressed. This paper evaluates two formulations of force-based frame element response sensitivity in numerical stability of the equations required to compute derivatives of the element response with respect to material, geometric, and member load properties under small deformations of a corotating reference frame. After a brief overview of the DDM's application to force-based elements, condition numbers associated with each formulation are derived to quantify the potential for round off errors. Perturbation analyses demonstrate the effect of an ill-conditioned matrix that is formed as part of one formulation, and a pushover analysis of a reinforced concrete column shows the evolution of 
each formulation's condition number as the column loses load carrying capacity.

\section{Governing Response Sensitivity Equations}

For nonlinear static finite-element analysis, the following system of equations must be assembled to solve for the nodal displacement sensitivity, $\partial \mathbf{U} / \partial \theta$

$$
\mathbf{K}_{T} \frac{\partial \mathbf{U}}{\partial \theta}=\frac{\partial \mathbf{P}}{\partial \theta}-\left.\frac{\partial \mathbf{P}_{r}}{\partial \theta}\right|_{\mathbf{U}}
$$

in which $\mathbf{K}_{T}=$ tangent stiffness matrix of the structure; $\partial \mathbf{P} / \partial \theta=$ derivative of the applied nodal load vector; and $\partial \mathbf{P}_{r} /\left.\partial \theta\right|_{\mathbf{U}}=$ conditional derivative of the resisting force vector, which is assembled from element contributions by standard finite-element procedures. Physically, the conditional derivative of $\mathbf{P}_{r}$ represents the nodal forces that must be applied to the structure to keep the nodal displacements, $\mathbf{U}$, fixed, while the state of the structure changes due to changes in the parameter $\theta$. The extension of Eq. (1) to nonlinear dynamic analysis is straightforward and its omission does not affect the evaluations presented.

The DDM is a two-phase process akin to the assembly and stress recovery phases of the ordinary finite-element response (Zhang and Der Kiureghian 1993). For frame finite elements formulated in end forces, $\mathbf{q}$, and deformations, $\mathbf{v}$, in a basic reference system free of rigid body displacement modes (Fig. 1), the conditional derivative of basic forces $\partial \mathbf{q} /\left.\partial \theta\right|_{\mathbf{v}}$, which has the same physical interpretation as $\partial \mathbf{P}_{r} /\left.\partial \theta\right|_{\mathbf{U}}$ but at the element level, must be transformed and assembled into the right-hand side of Eq. (1) during Phase I. After the solution of Eq. (1), the sensitivity of element deformations, $\partial \mathbf{v} / \partial \theta$, is obtained in Phase II by selection and transformation of entries in $\partial \mathbf{U} / \partial \theta$. The element deformation sensitivity is necessary to update constitutive models for path-dependent response and for recovering unconditional derivatives of the element response, e.g., forces or plastic rotations.

\section{Matrix Condition Numbers}

Much like the regular finite-element response, a stable solution to Eq. (1) depends on the condition number, $\kappa$, of the tangent stiffness matrix, $\mathbf{K}_{T}$. The condition number of a matrix is equal to the ratio of its largest to smallest singular values (Golub and Van Loan 1996;

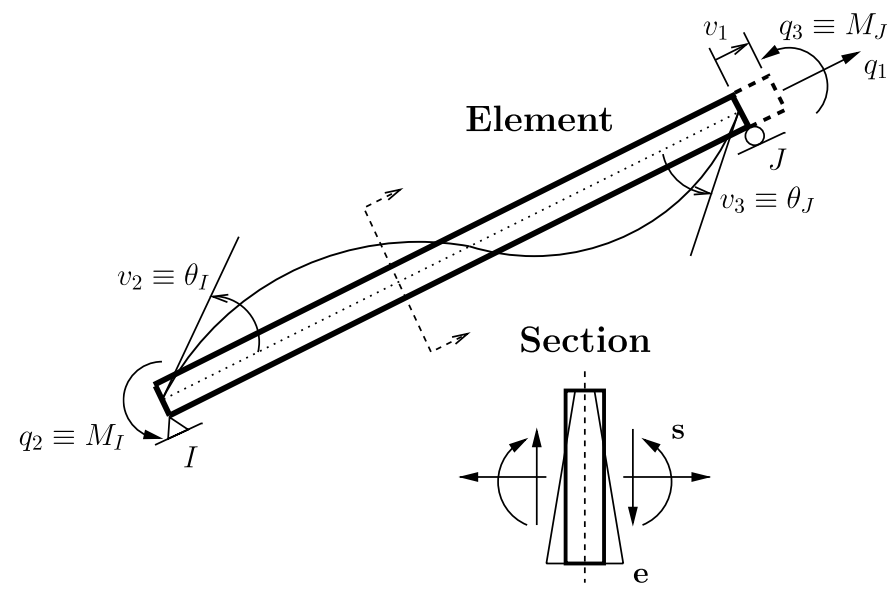

Fig. 1. Simply supported basic system for plane frame elements and section response quantities
Demmel 1997; Trefethen and Bau III 1997) or equivalently the product of maximum singular values of the matrix and its inverse

$$
\kappa(\mathbf{A})=\frac{\sigma_{\max }(\mathbf{A})}{\sigma_{\min }(\mathbf{A})}=\sigma_{\max }(\mathbf{A}) \sigma_{\max }\left(\mathbf{A}^{-1}\right)
$$

For a symmetric matrix, the condition number can be expressed in eigenvalues. A well-conditioned matrix has a low condition number $(\kappa \geq 1.0)$, whereas a singular matrix has $\kappa=\infty$. A matrix is said to be ill-conditioned if its condition number approaches $1 / \varepsilon_{\text {machine }}$, in which $\varepsilon_{\text {machine }}$ is machine precision in finite arithmetic.

For any linear system $\mathbf{A x}=\mathbf{b}$, the condition number of the coefficient matrix A provides a bound on how much the solution vector $\mathbf{x}$ will change with respect to perturbations of the right-hand side vector, $\mathbf{b}$

$$
\frac{\|\Delta \mathbf{x}\|}{\|\mathbf{x}\|} \leq \kappa(\mathbf{A}) \frac{\|\Delta \mathbf{b}\|}{\|\mathbf{b}\|}
$$

An alternative interpretation of this equation is that the condition number gives the maximum number of significant digits, $d$, lost in the solution to $\mathbf{A x}=\mathbf{b}$

$$
d \leq \log _{10} \kappa(\mathbf{A})
$$

The actual number of digits lost in the solution of realistic finiteelement problems is typically much less than that indicated by the condition number (Cook et al. 2002). Stiffness matrices assembled during nonlinear analysis of frames can be ill-conditioned because of differing units assigned to the nodal equations, distortions of the element mesh, the use of penalty functions to enforce multipoint constraints, the loss of an element's load carrying capacity, or the onset of geometric instability. The following sections develop the condition number associated with two DDM formulations of force-based element response sensitivity.

\section{Force-Based Response Sensitivity Equations}

To apply the DDM to force-based elements, it is necessary to differentiate the governing equations of equilibrium and compatibility with respect to an uncertain parameter of the structural model. The equations that govern force-based frame element response are summarized first, followed by their differentiation with respect to an uncertain model parameter.

\section{Governing Equations}

Force-based elements satisfy equilibrium of section and basic forces in strong form in which the homogeneous solution is given by the interpolation matrix, $\mathbf{b}(x)$

$$
\mathbf{s}(x)=\mathbf{b}(x) \mathbf{q}+\mathbf{s}_{p}(x)
$$

in which $\mathbf{q}=$ vector of basic forces; and $\mathbf{s}(x)$ contains the internal section forces along the element, as shown in Fig. 1 for a simply supported basic system. The vector $\mathbf{s}_{p}(x)$ represents the particular solution for section forces because of member loads applied in the basic system.

Compatibility between section and element deformations, $\mathbf{e}(x)$ and $\mathbf{v}$, respectively, is satisfied in integral form

$$
\mathbf{v}=\int_{0}^{L} \mathbf{b}^{T}(x) \mathbf{e}(x) d x
$$

For numerical implementation, this integral is evaluated over $N$ integration points, each with location $x_{i}$ and weight $w_{i}$, in the element domain $[0, L]$ 


$$
\mathbf{v}=\sum_{i=1}^{N} \mathbf{b}_{i}^{T} \mathbf{e}_{i} w_{i}
$$

in which evaluation of section response at the $i$ th integration point is abbreviated, e.g., $\mathbf{e}_{i} \equiv \mathbf{e}\left(x_{i}\right)$.

The partial derivative of the element deformations in Eq. (7) with respect to basic forces gives the element flexibility matrix

$$
\mathbf{f}=\frac{\partial \mathbf{v}}{\partial \mathbf{q}}=\sum_{i=1}^{N} \mathbf{b}_{i}^{T} \mathbf{f}_{s i} \mathbf{b}_{i} w_{i}
$$

in which $\mathbf{f}_{s i}=\partial \mathbf{e}_{i} / \partial \mathbf{s}_{i}=$ section flexibility matrix. The element flexibility matrix is inverted to obtain the basic stiffness matrix, $\mathbf{k}=\mathbf{f}^{-1}$, which is subsequently assembled into the tangent stiffness matrix of Eq. (1). The variational basis for force-based elements is described by Hjelmstad and Taciroglu (2005) and details on the numerical implementation of these elements within a displacement-driven finite-element framework are given by Neuenhofer and Filippou (1997).

\section{Differentiation of Governing Equations}

The equilibrium relationship in Eq. (5) is differentiated with respect to $\theta$, an uncertain parameter of the structural model

$$
\frac{\partial \mathbf{s}_{i}}{\partial \theta}=\mathbf{b}_{i} \frac{\partial \mathbf{q}}{\partial \theta}+\frac{\partial \mathbf{b}_{i}}{\partial \theta} \mathbf{q}+\frac{\partial \mathbf{s}_{p i}}{\partial \theta}
$$

The derivative of basic forces, $\partial \mathbf{q} / \partial \theta$, generally depends on every model parameter, whereas the derivative of the force interpolation matrix, $\partial \mathbf{b}_{i} / \partial \theta$, is generally nonzero for only geometric and numerical integration parameters, e.g., plastic hinge lengths, of the element. The derivative $\partial \mathbf{s}_{p i} / \partial \theta$ will be nonzero when $\theta$ represents a member load or geometric parameter of the element.

In addition, the compatibility equation of Eq. (7) is differentiated with respect to $\theta$

$$
\frac{\partial \mathbf{v}}{\partial \theta}=\sum_{i=1}^{N} \mathbf{b}_{i}^{T} \frac{\partial \mathbf{e}_{i}}{\partial \theta} w_{i}+\sum_{i=1}^{N} \frac{\partial \mathbf{b}_{i}^{T}}{\partial \theta} \mathbf{e}_{i} w_{i}+\sum_{i=1}^{N} \mathbf{b}_{i}^{T} \mathbf{e}_{i} \frac{\partial w_{i}}{\partial \theta}
$$

in which the last summation involving $\partial w_{i} / \partial \theta$ accounts for uncertain integration weights of the element.

The general form of the element constitutive relationship expresses the basic forces as a nonlinear function of the element deformations and the parameter, $\theta$, according to $\mathbf{q} \equiv \mathbf{q}(\mathbf{v}(\theta), \theta)$. Taking the derivative of this relationship with respect to $\theta$ gives

$$
\frac{\partial \mathbf{q}}{\partial \theta}=\mathbf{k} \frac{\partial \mathbf{v}}{\partial \theta}+\left.\frac{\partial \mathbf{q}}{\partial \theta}\right|_{\mathbf{v}}
$$

in which $\mathbf{k}=\partial \mathbf{q} / \partial \mathbf{v}=$ basic stiffness matrix.

At the constitutive level, the section forces, $\mathbf{s}$, at any location, $x$, along the element are expressed as a nonlinear function of the section deformation, e, and the parameter, $\theta$, through $\mathbf{s}(x) \equiv \mathbf{s}[x, \mathbf{e}(x, \theta), \theta]$. The derivative of this relationship at the $i$ th integration point is

$$
\frac{\partial \mathbf{s}_{i}}{\partial \theta}=\mathbf{k}_{s i} \frac{\partial \mathbf{e}_{i}}{\partial \theta}+\left.\frac{\partial \mathbf{s}_{i}}{\partial \theta}\right|_{\mathbf{e}_{i}}
$$

in which $\mathbf{k}_{s i}=\partial \mathbf{s}_{i} / \partial \mathbf{e}_{i}$ is the section stiffness matrix.

Two approaches to manipulating Eqs. (9)-(12) were developed by Scott et al. (2004) and Conte et al. (2004) to incorporate forcebased elements within a DDM-based framework for response sensitivity analysis. Both approaches were limited to the case in which $\theta$ represents a material parameter, where the derivatives $\partial \mathbf{b}_{i} / \partial \theta$ and $\partial w_{i} / \partial \theta$ are zero. To provide a complete evaluation of the numerical stability of each formulation, these derivatives are assumed to be nonzero.

\section{Consistent Formulation of Force-Based Response Sensitivity}

Scott et al. (2004) formulated response sensitivity equations for force-based elements by combining derivatives of the governing equations of equilibrium, compatibility, and constitution in a manner that is consistent with the element response. The equations summarized in this section provide a basis for numerical analysis of the inconsistent formulation presented in the following section and are used in the implementation of force-based DDM sensitivity in OpenSees (McKenna et al. 2000).

The derivation begins by solving for $\partial \mathbf{e}_{i} / \partial \theta$ in Eq. (12), then combining Eqs. (9) and (11) with this result

$$
\frac{\partial \mathbf{e}_{i}}{\partial \theta}=\mathbf{f}_{s i} \mathbf{b}_{i} \mathbf{k} \frac{\partial \mathbf{v}}{\partial \theta}+\mathbf{f}_{s i}\left(\left.\mathbf{b}_{i} \frac{\partial \mathbf{q}}{\partial \theta}\right|_{\mathbf{v}}+\frac{\partial \mathbf{b}_{i}}{\partial \theta} \mathbf{q}+\frac{\partial \mathbf{s}_{p i}}{\partial \theta}-\left.\frac{\partial \mathbf{s}_{i}}{\partial \theta}\right|_{\mathbf{e}_{i}}\right)
$$

This equation is used during Phase II to update the sensitivity of path-dependent section response, and it is required to find the conditional derivative of basic forces in Phase I. To this end, Eq. (13) is inserted in Eq. (10); then, with the definition of the element flexibility matrix in Eq. (8) and the identity $\mathbf{f k}=\mathbf{I}$, terms involving $\partial \mathbf{v} / \partial \theta$ cancel and the following expression is obtained

$$
\begin{aligned}
& \sum_{i=1}^{N} \mathbf{b}_{i}^{T} \mathbf{f}_{s i}\left(\left.\mathbf{b}_{i} \frac{\partial \mathbf{q}}{\partial \theta}\right|_{\mathbf{v}}+\frac{\partial \mathbf{b}_{i}}{\partial \theta} \mathbf{q}+\frac{\partial \mathbf{s}_{p i}}{\partial \theta}-\left.\frac{\partial \mathbf{s}_{i}}{\partial \theta}\right|_{\mathbf{e}_{i}}\right) w_{i} \\
& +\sum_{i=1}^{N}\left(\frac{\partial \mathbf{b}_{i}^{T}}{\partial \theta} \mathbf{e}_{i} w_{i}+\mathbf{b}_{i}^{T} \mathbf{e}_{i} \frac{\partial w_{i}}{\partial \theta}\right) \\
& =\mathbf{0}
\end{aligned}
$$

After inversion of the element flexibility matrix, the conditional derivative of the basic forces is

$$
\begin{aligned}
\left.\frac{\partial \mathbf{q}}{\partial \theta}\right|_{\mathbf{v}}= & \mathbf{k} \sum_{i=1}^{N} \mathbf{b}_{i}^{T} \mathbf{f}_{s i}\left(\left.\frac{\partial \mathbf{s}_{i}}{\partial \theta}\right|_{\mathbf{e}_{i}}-\frac{\partial \mathbf{b}_{i}}{\partial \theta} \mathbf{q}-\frac{\partial \mathbf{s}_{p i}}{\partial \theta}\right) w_{i} \\
& -\mathbf{k} \sum_{i=1}^{N}\left(\frac{\partial \mathbf{b}_{i}^{T}}{\partial \theta} \mathbf{e}_{i} w_{i}+\mathbf{b}_{i}^{T} \mathbf{e}_{i} \frac{\partial w_{i}}{\partial \theta}\right)
\end{aligned}
$$

which is assembled into the right-hand side of Eq. (1) during Phase I of DDM computations. For any one parameter, $\theta$, most terms on the right-hand side of this equation will be zero.

\section{Numerical Stability}

From Eqs. (13) and (15), it is observed that the numerical stability of solving for the conditional derivative of basic forces and the unconditional derivative of section deformations depends on the condition numbers of the basic stiffness matrix, $\mathbf{k}$, and section stiffness matrices, $\mathbf{k}_{s i}$. Condition numbers for the section stiffness matrices will be the same as those for the section flexibility matrices that appear in Eqs. (13) and (15). As a result, the numerical stability is related to the maximum condition number between the basic stiffness matrix and the section stiffness matrices

$$
\kappa_{f b}=\max \left[\kappa(\mathbf{k}), \max _{i=1}^{N} \kappa\left(\mathbf{k}_{s i}\right)\right]
$$

For a linear-elastic prismatic Bernoulli frame element of length $L$ in a simply supported basic system, the basic stiffness matrix is 


$$
\mathbf{k}=\left[\begin{array}{ccc}
E A / L & 0 & 0 \\
0 & 4 E I / L & 2 E I / L \\
0 & 2 E I / L & 4 E I / L
\end{array}\right]
$$

in which $E=$ elastic modulus; $A=$ cross-section area; and $I=$ second moment of the cross-section area. Because of matrix symmetry, the singular values of $\mathbf{k}$ are the same as its eigenvalues $E A / L, 2 E I / L$, and $6 E I / L$. The resulting condition number is

$$
\kappa(\mathbf{k})=\frac{\max (6 I, A)}{\min (2 I, A)}
$$

which can be expressed in the section radius of gyration, $r^{2}=I / A$. Similarly, the condition number of the section stiffness matrix, $\mathbf{k}_{s}=\operatorname{diag}[E A, \quad E I]$, whose singular values are $E A$ and $E I$, is

$$
\kappa\left(\mathbf{k}_{s}\right)=\frac{\max (I, A)}{\min (I, A)}
$$

which is equal to the radius of gyration if $r^{2}>1$ and $1 / r^{2}$ otherwise. Following Eq. (16), the union of these min / max expressions gives the condition number of the force-based formulation in the radius of gyration

$$
\kappa_{f b}= \begin{cases}1 / r^{2}, & r^{2} \leq 1 / 3 \\ 3, & 1 / 3<r^{2} \leq 1 / 2 \\ 6 r^{2}, & r^{2}>1 / 2\end{cases}
$$

This numerical analysis for a linear-elastic prismatic frame element represents the best case scenario, i.e., a lower bound on the condition number, for the response of a material nonlinear frame element. Condition numbers of the element and section stiffness matrices generally increase during a nonlinear analysis because $\sigma_{\min }$ tends to decrease rapidly, whereas $\sigma_{\max }$ stays relatively unchanged when plastic hinges form, concrete crushes, and strain hardening initiates (Lee and Filippou 2009).

\section{Inconsistent Formulation of Force-Based Response Sensitivity}

In the force-based response sensitivity formulation of Conte et al. (2004), an auxiliary element-level system of linear equations is formed to solve for derivatives of both the element and section response. The number of simultaneous equations depends on the number of integration points, $N$; the number of section forces, $N_{S R}$; and the number of element basic forces, $N_{B F}$.

The formulation begins by combining the derivatives of the section equilibrium and constitutive relationships of Eqs. (9) and (12), respectively

$$
\mathbf{k}_{s i} \frac{\partial \mathbf{e}_{i}}{\partial \theta}-\mathbf{b}_{i} \frac{\partial \mathbf{q}}{\partial \theta}=-\left.\frac{\partial \mathbf{s}_{i}}{\partial \theta}\right|_{\mathbf{e}_{i}}+\frac{\partial \mathbf{b}_{i}}{\partial \theta} \mathbf{q}+\frac{\partial \mathbf{s}_{p i}}{\partial \theta}
$$

The derivative of the element compatibility relationship in Eq. (10) is rewritten to isolate $\partial \mathbf{e}_{i} / \partial \theta$ on the left-hand side

$$
\sum_{i=1}^{N} \mathbf{b}_{i}^{T} \frac{\partial \mathbf{e}_{i}}{\partial \theta} w_{i}=\frac{\partial \mathbf{v}}{\partial \theta}-\sum_{i=1}^{N} \frac{\partial \mathbf{b}_{i}^{T}}{\partial \theta} \mathbf{e}_{i} w_{i}-\sum_{i=1}^{N} \mathbf{b}_{i}^{T} \mathbf{e}_{i} \frac{\partial w_{i}}{\partial \theta}
$$

The linear algebraic equations in Eqs. (21) and (22) can be written as a system of $N N_{S R}+N_{B F}$ simultaneous equations for unknown derivatives $\partial \mathbf{e}_{i} / \partial \theta$ and $\partial \mathbf{q} / \partial \theta$ of section deformations and basic forces, respectively

$$
\begin{array}{r}
{\left[\begin{array}{ccccc}
\mathbf{k}_{s 1} & \mathbf{0} & \ldots & \mathbf{0} & -\mathbf{b}_{1} \\
\mathbf{0} & \mathbf{k}_{s 2} & \ldots & \mathbf{0} & -\mathbf{b}_{2} \\
\vdots & \vdots & \ddots & \vdots & \vdots \\
\mathbf{0} & \mathbf{0} & \ldots & \mathbf{k}_{s N} & -\mathbf{b}_{N} \\
w_{1} \mathbf{b}_{1}^{T} & w_{2} \mathbf{b}_{2}^{T} & \ldots & w_{N} \mathbf{b}_{N}^{T} & \mathbf{0}
\end{array}\right]\left[\begin{array}{c}
\partial \mathbf{e}_{1} / \partial \theta \\
\partial \mathbf{e}_{2} / \partial \theta \\
\vdots \\
\partial \mathbf{e}_{N} / \partial \theta \\
\partial \mathbf{q} / \partial \theta
\end{array}\right]} \\
=\left[\begin{array}{c}
-\partial \mathbf{s}_{1} /\left.\partial \theta\right|_{\mathbf{e}_{1}}+\left(\partial \mathbf{b}_{1} / \partial \theta\right) \mathbf{q}+\partial \mathbf{s}_{p 1} / \partial \theta \\
-\partial \mathbf{s}_{2} /\left.\partial \theta\right|_{\mathbf{e}_{2}}+\left(\partial \mathbf{b}_{2} / \partial \theta\right) \mathbf{q}+\partial \mathbf{s}_{p 2} / \partial \theta \\
-\partial \mathbf{s}_{N} /\left.\partial \theta\right|_{\mathbf{e}_{N}}+\left(\partial \mathbf{b}_{N} / \partial \theta\right) \mathbf{q}+\partial \mathbf{s}_{p N} / \partial \theta \\
\partial \mathbf{v} / \partial \theta-\sum_{i=1}^{N}\left(\frac{\partial \mathbf{b}_{i}^{T}}{\partial \theta} \mathbf{e}_{i} w_{i}+\mathbf{b}_{i}^{T} \mathbf{e}_{i} \frac{\partial w_{i}}{\partial \theta}\right)
\end{array}\right]
\end{array}
$$

For notational convenience, this system of equations is denoted $\mathbf{S x}=\mathbf{p}$. During Phase I calculations, the term $\partial \mathbf{v} / \partial \theta$ in the right-hand side vector $\mathbf{p}$ is replaced by the zero vector so that the unknown vector $\mathbf{x}$ contains the sought after conditional derivative, $\partial \mathbf{q} /\left.\partial \theta\right|_{\mathbf{v}}$ in its last $N_{B F}$ rows. Similar to the right-hand side of Eq. (15), most terms in $\mathbf{p}$ will be zero for any one parameter of the structural model.

\section{Condition Number}

To assess the numerical stability of this sensitivity formulation, the condition number of the $\mathbf{S}$ matrix in Eq. (23) is computed from its singular values according to Eq. (2). For realistic physical properties, the trailing $N_{B F}$ rows and columns of $\mathbf{S}$ are close to zero relative to the section stiffness matrices on the diagonal, as observed in Fig. 2(a), in which the components of this matrix are plotted for a linear-elastic prismatic frame element with five Gauss-Lobatto integration points. As a result, the singular values of the section stiffness matrices will compose the largest singular values of $\mathbf{S}$

$$
\sigma_{\max }(\mathbf{S})=\max _{i=1}^{N} \sigma_{\max }\left(\mathbf{k}_{s i}\right)
$$

Similar inferences to determine the smallest singular value of $\mathbf{S}$ from Fig. 2(a) are not readily apparent, so the relationship $\sigma_{\max }\left(\mathbf{S}^{-1}\right)=1 / \sigma_{\min }(\mathbf{S})$ will be utilized. Closed-form inversion of $\mathbf{S}$ would be impractical; instead, the force-based response sensitivity equations from the consistent formulation described in the previous section will be employed for a direct solution to the inverse of $\mathbf{S}$.

The leading $N N_{S R}$ rows of $\mathbf{S}^{-1}$ are found by substituting Eq. (15) into Eq. (13) for each integration point

$$
\begin{aligned}
\frac{\partial \mathbf{e}_{i}}{\partial \theta}= & \mathbf{f}_{s i} \mathbf{b}_{i} \mathbf{k} \sum_{j=1}^{N} \mathbf{b}_{j}^{T} \mathbf{f}_{s j}\left(\left.\frac{\partial \mathbf{s}_{j}}{\partial \theta}\right|_{\mathbf{e}_{j}}-\frac{\partial \mathbf{b}_{j}}{\partial \theta} \mathbf{q}-\frac{\partial \mathbf{s}_{p j}}{\partial \theta}\right) w_{j} \\
& -\mathbf{f}_{s i}\left(\left.\frac{\partial \mathbf{s}_{i}}{\partial \theta}\right|_{\mathbf{e}_{i}}-\frac{\partial \mathbf{b}_{i}}{\partial \theta} \mathbf{q}-\frac{\partial \mathbf{s}_{p i}}{\partial \theta}\right) \\
& +\mathbf{f}_{s i} \mathbf{b}_{i} \mathbf{k}\left[\frac{\partial \mathbf{v}}{\partial \theta}-\sum_{i=1}^{N}\left(\frac{\partial \mathbf{b}_{i}^{T}}{\partial \theta} \mathbf{e}_{i} w_{i}-\mathbf{b}_{i}^{T} \mathbf{e}_{i} \frac{\partial w_{i}}{\partial \theta}\right)\right]
\end{aligned}
$$

Then, the trailing $N_{B F}$ rows of $\mathbf{S}^{-1}$ are found by substituting Eq. (15) into Eq. (11) 


$$
\frac{\partial \mathbf{q}}{\partial \theta}=\mathbf{k} \sum_{i=1}^{N} \mathbf{b}_{i}^{T} \mathbf{f}_{s i}\left(\left.\frac{\partial \mathbf{s}_{i}}{\partial \theta}\right|_{\mathbf{e}_{i}}-\frac{\partial \mathbf{b}_{i}}{\partial \theta} \mathbf{q}-\frac{\partial \mathbf{s}_{p i}}{\partial \theta}\right) w_{i}+\mathbf{k}\left[\frac{\partial \mathbf{v}}{\partial \theta}-\sum_{i=1}^{N}\left(\frac{\partial \mathbf{b}_{i}^{T}}{\partial \theta} \mathbf{e}_{i} w_{i}-\mathbf{b}_{i}^{T} \mathbf{e}_{i} \frac{\partial w_{i}}{\partial \theta}\right)\right]
$$

Writing out Eq. (25) for all $N$ integration points and then combining these equations with Eq. (26) gives a closed-form expression for $\mathbf{S}^{-1}$

$$
\mathbf{S}^{-1}=\left[\begin{array}{ccccc}
\mathbf{f}_{s 1}\left(\mathbf{I}-\mathbf{b}_{1} \mathbf{k} \mathbf{b}_{1}^{T} \mathbf{f}_{s 1} w_{1}\right) & -\mathbf{f}_{s 1} \mathbf{b}_{1} \mathbf{k} \mathbf{b}_{2}^{T} \mathbf{f}_{s 2} w_{2} & \ldots & -\mathbf{f}_{s 1} \mathbf{b}_{1} \mathbf{k} \mathbf{b}_{N}^{T} \mathbf{f}_{s N} w_{N} & \mathbf{f}_{s 1} \mathbf{b}_{1} \mathbf{k} \\
-\mathbf{f}_{s 2} \mathbf{b}_{2} \mathbf{k} \mathbf{b}_{1}^{T} \mathbf{f}_{s 1} w_{1} & \mathbf{f}_{s 2}\left(\mathbf{I}-\mathbf{b}_{2} \mathbf{k} \mathbf{b}_{2}^{T} \mathbf{f}_{s 2} w_{2}\right) & \ldots & -\mathbf{f}_{s 2} \mathbf{b}_{2} \mathbf{k} \mathbf{b}_{N}^{T} \mathbf{f}_{s N} w_{N} & \mathbf{f}_{s 2} \mathbf{b}_{2} \mathbf{k} \\
\vdots & \vdots & \ddots & \vdots & \vdots \\
-\mathbf{f}_{s N} \mathbf{b}_{N} \mathbf{k} \mathbf{b}_{1}^{T} \mathbf{f}_{s 1} w_{1} & -\mathbf{f}_{s N} \mathbf{b}_{N} \mathbf{k} \mathbf{b}_{2}^{T} \mathbf{f}_{s 2} w_{2} & \ldots & \mathbf{f}_{s N}\left(\mathbf{I}-\mathbf{b}_{N} \mathbf{k} \mathbf{b}_{N}^{T} \mathbf{f}_{s N} w_{N}\right) & \mathbf{f}_{s N} \mathbf{b}_{N} \mathbf{k} \\
-\mathbf{k} \mathbf{b}_{1}^{T} \mathbf{f}_{s 1} w_{1} & -\mathbf{k} \mathbf{b}_{2}^{T} \mathbf{f}_{s 2} w_{2} & \ldots & -\mathbf{k} \mathbf{b}_{N}^{T} \mathbf{f}_{s N} w_{N} & \mathbf{k}
\end{array}\right]
$$

in which $\mathbf{I}=N_{S R} \times N_{S R}$ identity matrix. Signs have been flipped on coefficients in the leading $N N_{S R}$ columns of $\mathbf{S}^{-1}$ for consistency with the right-hand side vector in Eq. (23).
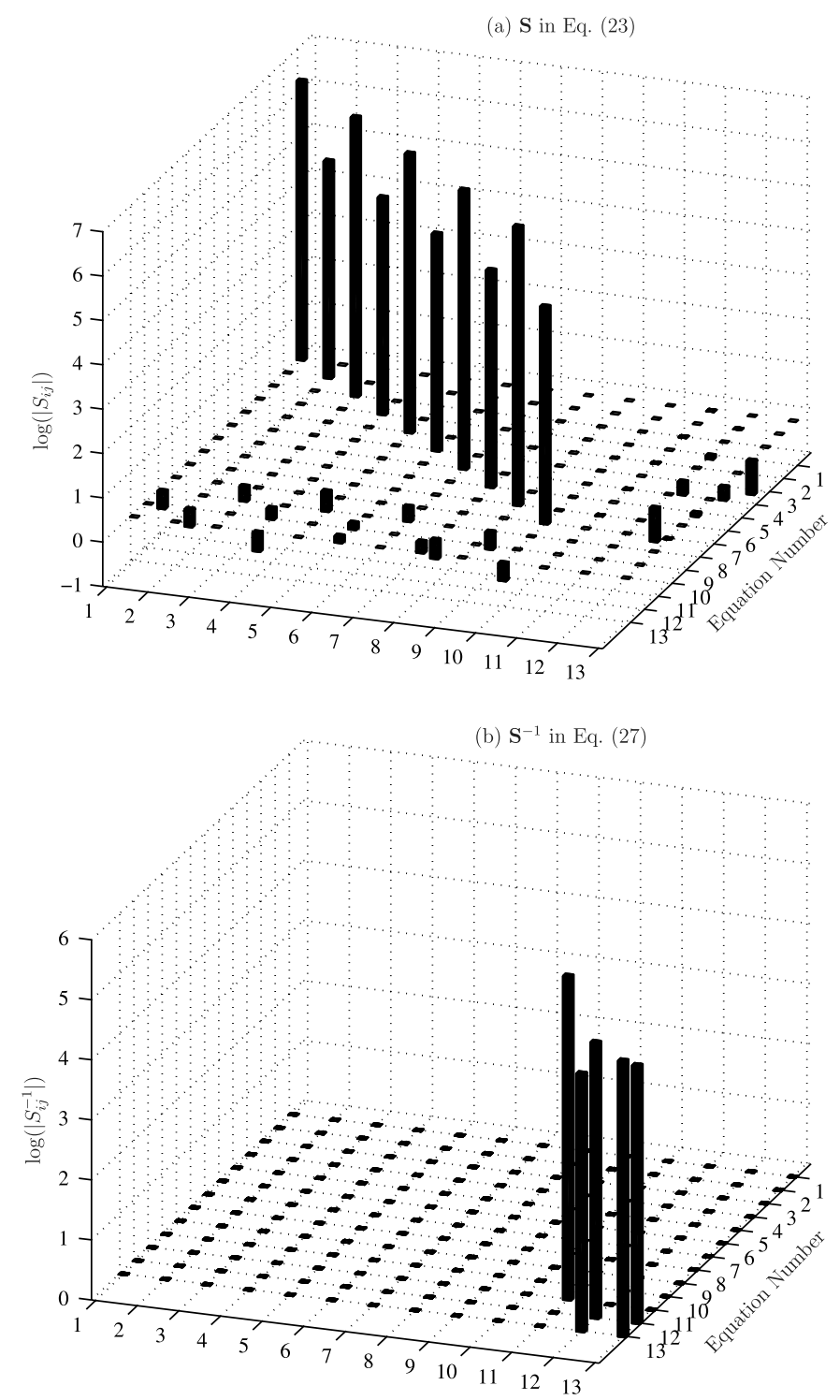

Fig. 2. Magnitude of matrix components for inconsistent response sensitivity formulation; linear-elastic prismatic frame element of length $L=8.0 \mathrm{~m}$ with section response determined at five Gauss-Lobatto points with $E=2.0 e 8 \mathrm{kPa}, A=0.01 \mathrm{~m}^{2}$, and $r^{2}=0.04 \mathrm{~m}^{2}$
It is observed that all block submatrices of $\mathbf{S}^{-1}$, except for the basic stiffness matrix, $\mathbf{k}$, on the last diagonal block, are on the order of either "flexibility" or "stiffness times flexibility," and thus several orders of magnitude less than the basic stiffness matrix for realistic physical properties. As a result, for realistic physical properties, the singular values of $\mathbf{k}$ will compose the largest singular values of $\mathbf{S}^{-1}$

$$
\sigma_{\max }\left(\mathbf{S}^{-1}\right)=\sigma_{\max }(\mathbf{k})
$$

This is confirmed in Fig. 2(b), in which the basic stiffness coefficients on the last block diagonal dominate the topology of $\mathbf{S}^{-1}$.

With the largest singular values of $\mathbf{S}$ and its inverse defined in section and basic stiffness matrices according to Eqs. (24) and (28), respectively, the condition number of $\mathbf{S}$ is

$$
\kappa(\mathbf{S})=\left[\max _{i=1}^{N} \sigma_{\max }\left(\mathbf{k}_{s i}\right)\right] \sigma_{\max }(\mathbf{k})
$$

For a linear-elastic prismatic Bernoulli frame element, this condition number can be expressed as a function of the section radius of gyration

$$
\kappa(\mathbf{S})= \begin{cases}\frac{(E A)^{2}}{L}, & r^{2} \leq 1 / 6 \\ \frac{6 E^{2} I A}{L}, & 1 / 6<r^{2} \leq 1 \\ \frac{6(E I)^{2}}{L}, & r^{2}>1\end{cases}
$$

As in the previous section, the condition number is developed here for a linear-elastic element to provide a lower bound on conditioning for material nonlinear analyses. The condition number dictated by Eq. (30) is plotted in Fig. 3 for a range of radii of gyration along with the condition number associated with the consistent sensitivity formulation [Eq. (20)]. For the selected physical properties of the simply supported basic system $(L=8.0 \mathrm{~m}, E=2.0 e 8 \mathrm{kPa}$, and $A=0.01 \mathrm{~m}^{2}$ ), Fig. 3 indicates that the inconsistent formulation of force-based response sensitivity analysis can lose up to 13 significant digits because of round off error.

The ill-conditioning of the inconsistent formulation can be mitigated by viewing Eqs. (21) and (22) as an equality constrained least-squares problem. To this end, Eq. (21) represents an underdetermined system whose solution is minimized subject to the constraints of Eq. (22). As shown in Appendix I, solution of these equations through the nullspace method (Bjorck 1996; Golub and Van Loan 1996) results in a condition number on the order of $E$ instead of the $E^{2}$ indicated in Eq. (30). The following perturbation analysis shows the effects of ill-conditioning in forming and solving the square system of equations in Eq. (23), which was the approach proposed in Conte et al. (2004). 


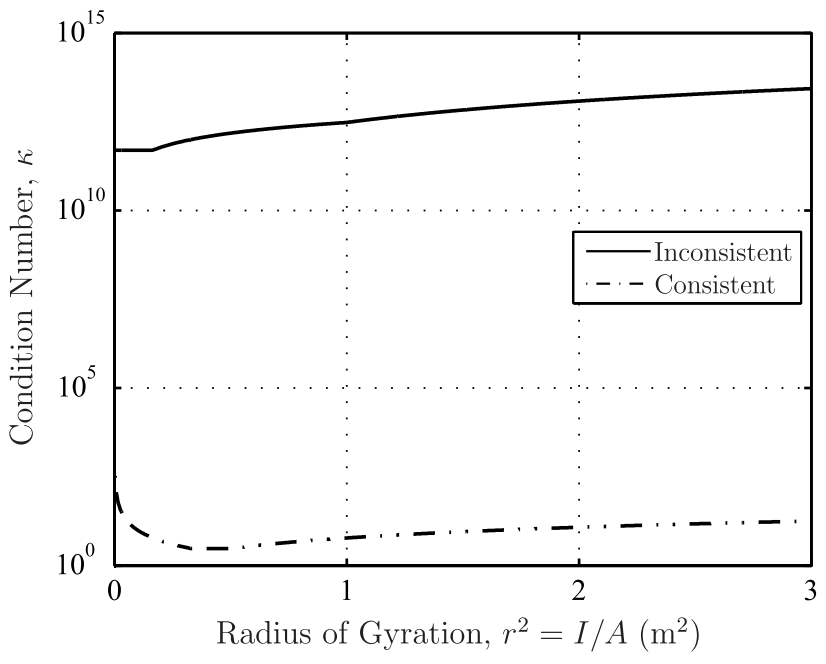

Fig. 3. Condition number of consistent and inconsistent force-based response sensitivity formulations for a linear-elastic prismatic frame element with varying radius of gyration and $E=2.0 e 8 \mathrm{kPa}$, $A=0.01 \mathrm{~m}^{2}$, and $L=8.0 \mathrm{~m}$

\section{Perturbation Analysis}

To demonstrate the actual effects of an ill-conditioned $\mathbf{S}$ matrix in solving the system of Eq. (23), the right-hand side vector is formed for a parameter, $\theta$, of a single element in the simply supported basic system. The physical properties are $L=8.0 \mathrm{~m}, E=2.0 e 8 \mathrm{kPa}$, and $A=0.01 \mathrm{~m}^{2}$; and the radius of gyration is set to $r^{2}=$ $0.04 \mathrm{~m}^{2}$, making the condition number of $\mathbf{S}$ equal to $5.0 e 11$ according to Eq. (30). A uniformly distributed load of $15 \mathrm{kN} / \mathrm{m}$ is applied so that member loads are included in the analysis.

For any right-hand side and solution vectors obtained through Eq. (23), the actual number of digits lost is computed according to Eqs. (3) and (4) for unit perturbations of each equation

$$
d_{j}=\left\lceil\log \left(\frac{\left\|\Delta \mathbf{x}_{j}\right\|}{\|\mathbf{x}\|}\|\mathbf{p}\|\right)\right\rceil
$$

in which $\mathbf{p}$ and $\mathbf{x}=$ right-hand side and unknown vectors, respectively, in Eq. (23). The vector $\Delta \mathbf{x}_{j}$ is the solution to $\mathbf{S} \Delta \mathbf{x}_{j}=\Delta \mathbf{p}_{j}$, in which $\Delta \mathbf{p}_{j}$ is all zero except for a one in the $j$ th row.

The response sensitivity is computed with respect to the element length, making $\partial L / \partial \theta \neq 0$ so that all rows of the right-hand side vector in Eq. (23) will be nonzero during Phase I and Phase II calculations. The resulting number of digits lost is plotted in Fig. 4, in which it is seen that the solution vector is relatively insensitive to perturbations in the leading $N N_{S R}$ entries of $\mathbf{p}$, i.e., to perturbations in the derivatives of section-level response. On the other hand, the solution is extremely sensitive to perturbations of element-level derivatives in the last $N_{B F}$ rows of $\mathbf{p}$, in which a unit change in the right-hand side leads to a relative change of $2.831 \times 10^{5}$ in magnitude of the solution vector. According to Eq. (31), six digits are lost because of perturbations of element-level derivatives in the right-hand side of Eq. (23), effectively precluding the inconsistent formulation from single precision arithmetic in which only seven digits of precision are available (IEEE 2008).

In the case in which $\theta$ represents a section constitutive parameter, results nearly identical to those shown in Fig. 4 are obtained. During Phase I calculations, the final $N_{B F}$ rows of the right-hand side vector are identically equal to zero and thus not subject to any round off errors. During Phase II calculations, however, round off

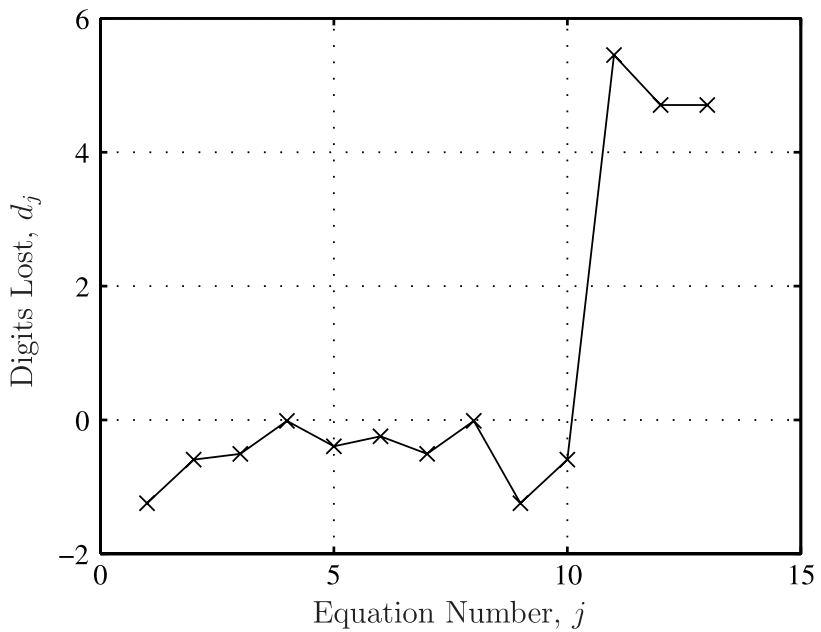

Fig. 4. Digits lost in inconsistent force-based response sensitivity formulation because of perturbations of each equation in the linear system of Eq. (23); frame element $\left(N_{S R}=2, N_{B F}=3\right)$ with $N=5$ integration points

errors in the global solution of Eq. (1) can lead to relatively large errors in recovering the unconditional derivatives, $\partial \mathbf{e}_{i} / \partial \theta$ and $\partial \mathbf{q} / \partial \theta$, through the solution to Eq. (23) at the element level. This is an important consideration for path-dependent nonlinear analyses in which the derivative of section deformations, $\partial \mathbf{e}_{i} / \partial \theta$, depends on all parameters of the structural model, even those that do not belong to the element. Thus, the results shown in Fig. 4 are an unbiased presentation of the numerical instability inherent in the sensitivity formulation of Conte et al. (2004) for uncertain material and geometric parameters of a structural model.

\section{Reinforced Concrete Column}

To demonstrate further the ill-conditioning of the inconsistent response sensitivity formulation, the load-displacement response of a reinforced concrete column is computed and the evolution of condition numbers is tracked. The structural model is on the basis of specimen 7 in the tests of Tanaka and Park (1990) and is chosen because of its observed loss of load carrying capacity. Column dimensions and cross-section details are shown in Fig. 5. It is anticipated that as a plastic hinge forms during the pushover analysis, the section stiffness of the hinge region will approach singularity and affect the conditioning of the element response.

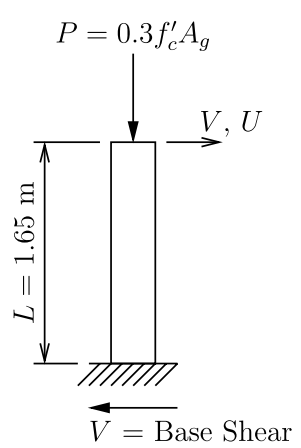

$550 \mathrm{~mm} \times 550 \mathrm{~mm}$ square

12 bars, $d_{b}=20 \mathrm{~mm}$ $40 \mathrm{~mm}$ clear cover

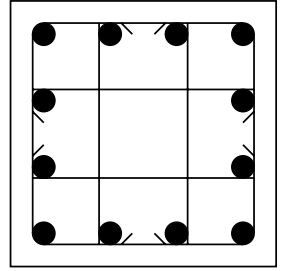

Tanaka and Park (1990) Specimen 7

Fig. 5. Geometry and reinforcing details of reinforced concrete column example 

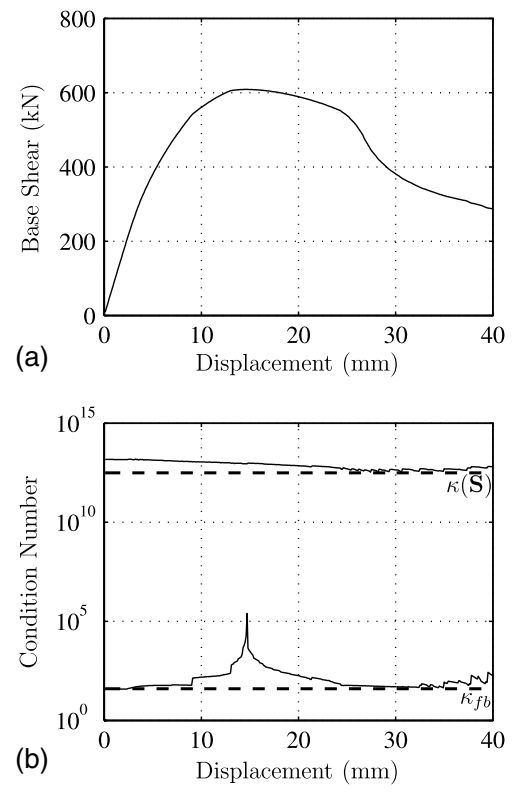

Fig. 6. Reinforced concrete column example: (a) load-displacement response; (b) condition number of force-based element response and inconsistent response sensitivity formulation

The column response is computed by using one force-based frame element with four Gauss-Lobatto integration points. Fiber discretization of the cross section captures the effects of axialmoment interaction. Concrete fibers have a compressive strength of $f_{c}^{\prime}=32 \mathrm{MPa}$ with confining effects determined according to Mander et al. (1988), whereas the reinforcing steel is bilinear with elastic modulus $E=200,000 \mathrm{MPa}$; yield stress $f_{y}=510 \mathrm{MPa}$; and $1 \%$ strain hardening. A compressive axial load equal to $3,540 \mathrm{kN}$ ( $30 \%$ of the gross section capacity) is held constant during the pushover analysis. The cross section is $550 \mathrm{~mm}^{2}$, giving a radius of gyration $r^{2}=0.02521 \mathrm{~m}^{2}$. By using the gross section properties and cracked concrete modulus, lower bounds on the condition numbers associated with the force-based element and the inconsistent response sensitivity formulation are computed as 39.67 and $3.120 \times 10^{12}$, respectively, according to Eqs. (20) and (30).

The load-displacement response of the column under a displacement-controlled analysis in OpenSees (McKenna et al. 2000) is shown in Fig. 6(b), in which peak lateral capacity is reached at approximately $15-\mathrm{mm}$ lateral displacement. The stiffness matrix at each of the four Gauss-Lobatto points is recorded at every time step to form the $\mathbf{S}$ matrix and compute its condition number along with the condition number associated with the underlying force-based element response, both by using the condition number function, cond(), in MATLAB 7.11.

As the column loses stiffness during the pushover analysis because of the formation of a plastic hinge at its base, the magnitude of the force-based formulation condition number spikes to $2.515 \times 10^{5}$ then returns to the neighborhood of its estimated lower bound (39.67), as shown in Fig. 6(b). This condition number remains several orders of magnitude less than that for the inconsistent sensitivity formulation, which ranges from $3.396 \times 10^{12}$ to $1.535 \times$ $10^{13}$ (lower bound is $3.120 \times 10^{12}$ ). A spike is not observed in the history of $\kappa(\mathbf{S})$ because it is the product of maximum singular values of the basic and section stiffness matrices, instead of the ratio of maximum to minimum singular values of these matrices.

\section{Conclusions}

The DDM maintains numerical consistency with the underlying finite-element response; however, this is not the case if the elementlevel response sensitivity solution is ill-conditioned. This was shown for a force-based element response sensitivity formulation (Conte et al. 2004) in which the associated condition number is equal to the product of the largest singular values of the basic and section stiffness matrices, whereas the conditioning of the underlying force-based element response depends only on the ratio of singular values of these matrices. The circumstances under which numerical instabilities can arise in the incosistent formulation depend on the right-hand side vector of Eq. (23) and are summarized as follows:

- When the parameter $\theta$ corresponds to an uncertain section-level constitutive property or member load, no ill-conditioning of the system in Eq. (23) during Phase I occurs because all terms in the trailing $N_{B F}$ rows of the right-hand side vector are zero.

- When $\theta$ corresponds to an uncertain nodal coordinate at the element ends or an element integration parameter, e.g., plastic hinge length, the system in Eq. (23) is ill-conditioned during Phase I because the terms $\partial \mathbf{b}_{i} / \partial \theta$ and $\partial w_{i} / \partial \theta$ are nonzero in the trailing $N_{B F}$ rows of the right-hand side vector in this case.

- The system in Eq. (23) is always ill-conditioned during Phase II because the element deformation sensitivity, $\partial \mathbf{v} / \partial \theta$, is nonzero in general for all types of parameters when the constitutive response is path dependent.

Thus, the inconsistent formulation provides well-conditioned results during Phase I for only section-level parameters owing to constitutive properties or member loads. Because of its consistency with the element response, these numerical issues are not present in the force-based element response sensitivity formulation developed by Scott et al. (2004), for which well-conditioned results are attained during both Phases I and II for all types of uncertain material, geometric, or load parameters. Similar numerical analyses for other finite-element formulations and constitutive models should be carried out to ensure consistency is preserved for the reliability, optimization, and system identification applications that rely on the accurate and efficient computation of response sensitivity to converge to an optimal solution point.

\section{Appendix I. Equality Constrained Least-Squares Approach}

An equality constrained least-squares problem is posed as

$$
\min \|\mathbf{A x}-\mathbf{b}\|_{2} \quad \text { subject to } \mathbf{B} \mathbf{x}=\mathbf{d}
$$

in which $\mathbf{A x}=\mathbf{b}=$ system of underdetermined equations; and $\mathbf{B} \mathbf{x}=\mathbf{d}=$ system of linear constrain equations. In the context of the inconsistent response sensitivity formulation, $\mathbf{A}$ and $\mathbf{b}$ represent the $N N_{S R}$ equations in Eq. (21), whereas $\mathbf{B}$ and $\mathbf{d}$ encompass the $N_{B F}$ equations in Eq. (22), which can each be extracted from the leading $N N_{S R}$ and trailing $N_{B F}$ rows of the matrix-vector system in Eq. (23).

The nullspace method (Bjorck 1996; Golub and Van Loan 1996) uses $Q R$ factorization to reformulate a constrained least-squares problem into an equivalent unconstrained problem. The first step in the nullspace method is to find the full $Q R$ factorization of $\mathbf{B}^{T}$, as defined in Eq. (22)

$$
\mathbf{B}^{T}=\mathbf{Q}\left[\begin{array}{c}
\mathbf{R} \\
\mathbf{0}
\end{array}\right]
$$


in which $\mathbf{Q}=N_{\text {eqn }} \times N_{\text {eqn }}$; and $\mathbf{R}=N_{B F} \times N_{B F}$. Then, the columns of the A matrix defined in Eq. (21) are rotated by the orthogonal matrix $\mathbf{Q}$ and partitioned

$$
\mathbf{A} \mathbf{Q}=\left[\begin{array}{ll}
\mathbf{A}_{1} & \mathbf{A}_{2}
\end{array}\right]
$$

in which $\mathbf{A}_{1}=N N_{S R} \times N_{B F}$; and $\mathbf{A}_{2}=N N_{S R} \times N N_{S R}$. With this transformed system, the solution for $\mathbf{x}$ is

$$
\mathbf{x}=\mathbf{Q}\left[\begin{array}{l}
\mathbf{y} \\
\mathbf{z}
\end{array}\right]
$$

in which $\mathbf{y}$ is found by linear solution with the upper-triangular $\mathbf{R}$ matrix

$$
\mathbf{R}^{T} \mathbf{y}=\mathbf{d}
$$

in which $\mathbf{R}$ has the same condition number as $\mathbf{B}$ after the $Q R$ factorization. It is also noted that $\mathbf{y}=\mathbf{d}=\mathbf{0}$ in the conditional derivative case for Phase I calculations.

Because of a sufficient number of constraint equations, the lower dimension least-squares problem of the nullspace method

$$
\min \left\|\mathbf{A}_{2} \mathbf{z}-\left(\mathbf{b}-\mathbf{A}_{1} \mathbf{y}\right)\right\|_{2}
$$

can be made zero, giving a second system of $N N_{S R}$ linear equations for $\mathbf{z}$

$$
\mathbf{A}_{2} \mathbf{z}=\mathbf{b}-\mathbf{A}_{1} \mathbf{y}
$$

The $\mathbf{y}$ and $\mathbf{z}$ vectors are then transformed back to the original space according to Eq. (35).

The stability of the nullspace method depends on the condition numbers of $\mathbf{B}$ and $\mathbf{A}_{2}$, owing to the solution to the linear systems in Eqs. (36) and (38) (Bjorck 1996). The condition number $\kappa(\mathbf{B})$ is on the order of the element length, whereas $\kappa\left(\mathbf{A}_{2}\right)$ is on the order of the largest singular value of all section stiffness matrices, i.e.

$$
\kappa\left(\mathbf{A}_{2}\right) \approx \max _{i=1}^{N} \sigma_{\max }\left(\mathbf{k}_{s i}\right)
$$

For a linear-elastic prismatic frame element, this gives the condition number

$$
\kappa\left(\mathbf{A}_{2}\right) \approx \max (E A, E I)
$$

Although this is a more stable approach than solving the linear system of equations in Eq. (23), the condition number still depends on the elastic modulus, $E$, as opposed to $E^{2}$.

\section{Acknowledgments}

The writer would like to thank Professor Michele Barbato of Louisiana State University and Professor Joel Conte of University of California, San Diego for fruitful communication regarding the force-based element response sensitivity formulations presented in this paper.

\section{Notation}

The following symbols are used in this paper:

$\mathbf{b}=$ section force interpolation matrix;

$\mathbf{e}=$ section deformation vector;

$\mathbf{f}=$ element flexibility matrix;

$\mathbf{f}_{s}=$ section flexibility matrix;

$\mathbf{K}_{T}=$ structural tangent stiffness matrix;

$\mathbf{k}=$ element basic stiffness matrix; $\mathbf{k}_{s}=$ section stiffness matrix;

$\mathbf{P}=$ structural load vector;

$\mathbf{P}_{r}=$ structural resisting force vector;

$\mathbf{q}=$ element basic force vector;

$\mathbf{s}=$ section force vector;

$\mathbf{S}_{p}=$ section force vector because of member loads;

$\mathbf{U}=$ structural displacement vector;

$\mathbf{v}=$ element deformation vector;

$w=$ element integration weight;

$\kappa=$ matrix condition number; and

$\sigma=$ matrix singular value.

\section{References}

Barbato, M., and Conte, J. P. (2006). "Finite element structural response sensitivity and reliability analyses using smooth versus non-smooth material constitutive models." Int. J. Reliab. Saf., 1(1/2), 3-39.

Bebamzadeh, A., and Haukaas, T. (2008). "Second-order sensitivities of inelastic finite-element response by direct differentiation." J. Eng. Mech., 134(10), 867-880.

Bjorck, A. (1996). Numerical methods for least squares problems, SIAM, Philadelphia.

Choi, K. K., and Santos, J. L. T. (1987). "Design sensitivity analysis of nonlinear structural systems, Part I: Theory." Int. J. Numer. Methods Eng., 24(11), 2039-2055.

Conte, J. P., Barbato, M., and Spacone, E. (2004). "Finite element response sensitivity analysis using force-based frame models." Int. J. Numer. Methods Eng., 59(13), 1781-1820.

Conte, J. P., Vijalapura, P. K., and Meghalla, M. (2003). "Consistent finite-element response sensitivity analysis." J. Eng. Mech., 129(12), 1380-1393.

Cook, R. D., Malkus, D. S., Plesha, M. E., and Witt, R. J. (2002). Concepts and applications of finite element analysis, 4th Ed., Wiley, New York.

Demmel, J. W. (1997). Applied numerical linear algebra, SIAM, Philadelphia.

Fellin, W., and Ostermann, A. (2006). "Parameter sensitivity in finite element analysis with constitutive models of the rate type." Int. J. Numer. Anal. Methods Geomech., 30(2), 91-112.

Golub, G. H., and van Loan, C. F. (1996). Matrix computations, 3rd Ed., Johns Hopkins Univ. Press, Baltimore, MD.

Gong, Y., Xu, L., and Grierson, D. E. (2006). "Sensitivity analysis of steel moment frames accounting for geometric and material nonlinearity." Comput. Struct., 84(7), 462-475.

Gu, Q., Barbato, M., and Conte, J. P. (2009). "Handling of constraints in finite-element response sensitivity analysis." J. Eng. Mech., 135(12), $1427-1438$.

Habibi, A., and Moharrami, H. (2010). "Nonlinear sensitivity analysis of reinforced concrete frames." Finite Elem. Anal. Des., 46(7), 571-584.

Haukaas, T. (2006). "Efficient computation of response sensitivities for inelastic structures." J. Struct. Eng., 132(2), 260-266.

Hjelmstad, K. D., and Taciroglu, E. (2005). "Variational basis of nonlinear flexibility methods for structural analysis of frames." J. Eng. Mech., 131(11), 1157-1169.

IEEE. (2008). IEEE standard for floating point arithmetic (754-2008), Piscataway, NJ.

Kleiber, M., Antunez, H., Hien, T. D., and Kowalczyk, P. (1997). Parameter sensitivity in nonlinear mechanics, Wiley, New York.

Lee, C.-L., and Filippou, F. C. (2009). "Frame elements with mixed formulation for singular section response." Int. J. Numer. Methods Eng., 78(11), 1320-1344.

Mander, J. B., Priestley, M. J. N., and Park, R. (1988). "Theoretical stress-strain model for confined concrete." J. Struct. Eng., 114(8), 1804-1826.

MATLAB 7.11 [Computer software]. MathWorks, Natick, MA.

McKenna, F., Fenves, G. L., and Scott, M. H. (2000). Open system for earthquake engineering simulation, Univ. of California, Berkeley, CA.

Neuenhofer, A., and Filippou, F. C. (1997). "Evaluation of nonlinear frame finite-element models." J. Struct. Eng., 123(7), 958-966. 
Pajot, J. M., and Maute, K. (2006). "Analytical sensitivity analysis of geometrically nonlinear structures based on the co-rotational finite element method." Finite Elem. Anal. Des., 42(10), 900-913.

Scott, M. H., and Filippou, F. C. (2007). "Exact response gradients for large displacement nonlinear beam-column elements." J. Struct. Eng., 133(2), 155-165.

Scott, M. H., Franchin, P., Fenves, G. L., and Filippou, F. C. (2004). "Response sensitivity for nonlinear beam-column elements." J. Struct. Eng., 130(9), 1281-1288.

Tanaka, H., and Park, R. (1990). "Effect of lateral confining reinforcement on the ductile behaviour of reinforced concrete columns." Rep. No. 90-2, Dept. of Civil Engineering, Univ. of Canterbury,
Christchurch, NZ.

Trefethen, L. N., and Bau, D. III. (1997). Numerical linear algebra, SIAM, Philadelphia.

Tsay, J. J., and Arora, J. S. (1990). "Nonlinear structural design sensitivity analysis for path dependent problems. Part I: General theory." Comput. Methods Appl. Mech. Eng., 81(2), 183-208.

Zhang, Y., and Der Kiureghian, A. (1993). "Dynamic response sensitivity of inelastic structures." Comput. Methods Appl. Mech. Eng., 108(1-2), $23-36$.

Zona, A., Barbato, M., and Conte, J. P. (2005). "Finite element response sensitivity analysis of steel-concrete composite beams with deformable shear connection." J. Eng. Mech., 131(11), 1126-1139. 